\title{
Risk stratification by CMR in Chagas' disease
}

\author{
Marly M Uellendahl \\ From 2011 SCMR/Euro CMR Joint Scientific Sessions \\ Nice, France. 3-6 February 2011
}

Chagas' disease $(C D)$ still remains an important health problem in Latin America. Cardiac involvement in $C D$ is associated wiht a high mortality and morbidity and one of the major challenges is to identify high risk patients. Therefore a risk score to predict death in CD was recently developed and validated by Rassi and colleagues. Considering the hole of myocardial fibrosis(MF) on the pathogenesis of $\mathrm{CD}$ we believe that $\mathrm{CMR}$ (cardiovascular magnetic resonance) may become an important method to stratify patients with $\mathrm{CD}$. We sought to evaluate morphological and functional characteristics, as well as the extent of MF in patients with $\mathrm{CD}$ by CMR and to search the prognostic value of MF detected by CMR, comparing these findings with the recently developed score of mortality (Rassi's score).Thirty-nine seropositive patients for CD were studied , divided in two main groups: group I, without apparent cardiomyopathy consisted of 11 asymptomatic patients without signs of cardiac involvement, and group II, with apparent cardiomyopathy, consisted of 28 patients with known heart involvement. We excluded patients with previous myocardial infarction, clinical or laboratory evidence of ischemic heart disease, increased cholesterol levels, diabetes, heart valve disease, pacemakers, Implantable deffibrilators and vascular brain clips. All patients underwent CMR examination on 1,5T GE echo-speed, SYSTEM (Wakeusha, Wiscosin). Short and long-axis of the heart were obtained during breath-hold and triggered on eletrocardiogram pulse sequences. The first sequence was a gradient-echo (steady-sate free procession) to assess left ventricular (LV) and right ventricular (RV) morphology and function. The second sequence was an inversionrecovery prepared gradient-echo to obtain myocardial delayed enhancement (MDE), 10 to 20 minutes after intravenous bolus of $0.2 \mathrm{mmol} / \mathrm{Kg}$ of gadolinium-based contrast. Regarding the morphological and functional analysis, significant differences were observed in both groups $(\mathrm{p}<0.001)$. Our data supports that there is an inverse correlation between increasing Rassi's score

Heart Institute (InCor-FMUSP) - São Paulo University, São Paulo, Brazil

numbers and LV ejection fraction $(\mathrm{r}=-0.78 \mathrm{p}<0.001)$. Furthermore there was a strong correlation with the extent of MF and the Rassi's score $(r=0.76, p<0.001)$; and also with Rassi's risk groups (low, intermediate and high risk) $(5 \pm 7.7 ; 19.8 \pm 17.3$; e $38.1 \pm 17.7 \%$, respectively ( $\mathrm{p}<0,001$, ANOVA). We concluded that CMR is an important technique in the evaluation of patients with $\mathrm{CD}$, stressing morphological and functional differences in all clinical presentations. The strong correlation with the Rassi's score and the extent of myocardial fibrosis detected by CMR, emphasizes its role in the prognostic stratification of patients with CD.

Published: 2 February 2011

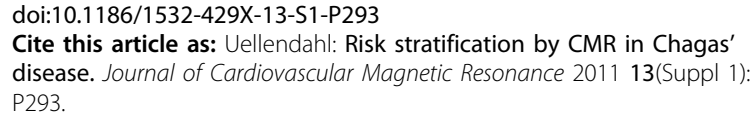

Submit your next manuscript to BioMed Central and take full advantage of:

- Convenient online submission

- Thorough peer review

- No space constraints or color figure charges

- Immediate publication on acceptance

- Inclusion in PubMed, CAS, Scopus and Google Scholar

- Research which is freely available for redistribution
C 2011 Uellendahl; licensee BioMed Central Ltd. This is an open access article distributed under the terms of the Creative Commons Attribution License (http://creativecommons.org/licenses/by/2.0), which permits unrestricted use, distribution, and reproduction in any medium, provided the original work is properly cited. 